\title{
Advances in Technology and Application of Silicon Drift Detectors
}

Thomas Schülein, Ralf Terborg, Martin Rohde

RÖNTEC AG, Schwarzschildstr. 12, D-12489 Berlin, Germany

In the past few years, Silicon Drift Detectors (SDD) have become more and more common in the field of X-ray detection. Since the manufacturing process and the functional principle of SDD differ from those of other solid state detectors, some properties of these detectors are different too.

One of the remarkable properties of the SDD (Fig. 1) is its extraordinary count-rate capability of up to one million counts per second at good or moderate energy resolution. However, the overall spectral performance was complained about and said to prevent accurate quantitative analysis. It was therefore considered chiefly an ideal device for high speed mapping and element imaging.

The recently developed Silicon Drift Detector Droplet ( $\mathrm{SD}^{3}$, Fig. 2), matches or even outperforms the classic $\mathrm{Si}(\mathrm{Li})$ spectrometer in terms of both energy resolution and count rate (Fig. 3). The somewhat higher tail and shelf effects of SDD and $\mathrm{SD}^{3}$ compared to most advanced $\mathrm{Si}(\mathrm{Li})$ detectors are meanwhile well understood [1,2,3]. Detailed investigation and physical modelling led to technological advances and improved correction. In addition, improvements have been achieved with regard to detector efficiency and solid angle. With $500 \mu \mathrm{m}$ thickness and active detector areas of 10,20 or even $30 \mathrm{~mm}^{2}$ the new SDD overcome the limitations of former generation devices, which were $300 \mu \mathrm{m}$ thick and had an active area of only $5 \mathrm{~mm}^{2}$.

Altogether, advanced SDD and $\mathrm{SD}^{3}$ spectrometers are well suited for qualitative and quantitative analysis, even in the range of light elements, thus breaking the above paradigm.

Besides the development of advanced SDD, the trend of building multiple element detectors is another approach to further enlarging the solid angle. Multiple element detectors exhibit an improved count-rate capability, since the specially adapted multi-channel electronics allows parallel signal processing. This way the effective detection area and total count-rate capability is multiplied by the number of detector channels. The flexibility of the geometrical design allows for various structures, such as cloverleaf-like 4 channel detectors or 12 channel ring shaped detectors (Fig. 4).

It's mainly the most modern analytical approaches, like spectral imaging or combined EDX/EBSD, that take particular advantage of both, the good spectral properties and the extraordinary count rate capabilities of advanced SDD. Especially with multi-channel SDD spectral imaging becomes a method for routine, everyday use, because it can be performed within only a fraction of the time required with conventional detector technology.

\section{References}

[1] R. Terborg, M. Rohde, Microsc. Microanal. 9 (Suppl.2) Proceedings (2003), 120

[2] R. Hartmann et al., Nucl. Instr. and Meth. A 377 (1996) 191

[3] F. Scholze, M. Procop, X-ray Spectrom. 30 (2001) 69 


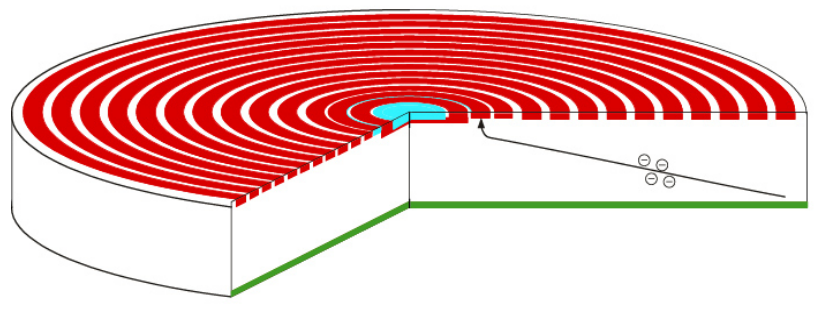

FIG. 1. Design of a Silicon Drift Detector

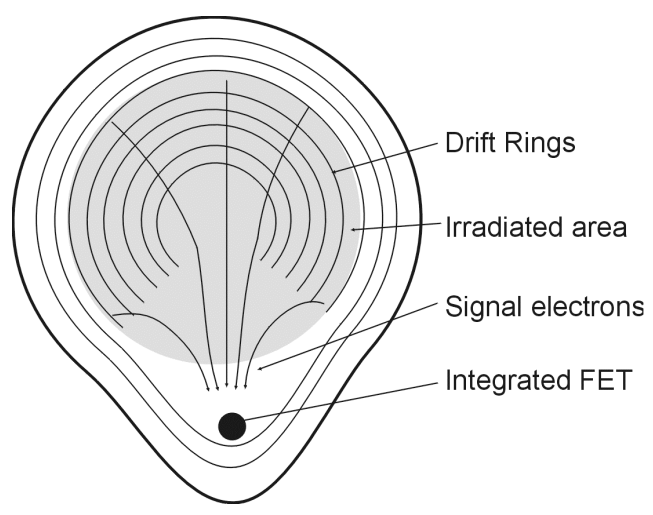

FIG. 2. Modified design of a Silicon Drift Detector Droplet $\left(\mathrm{SD}^{3}\right)$

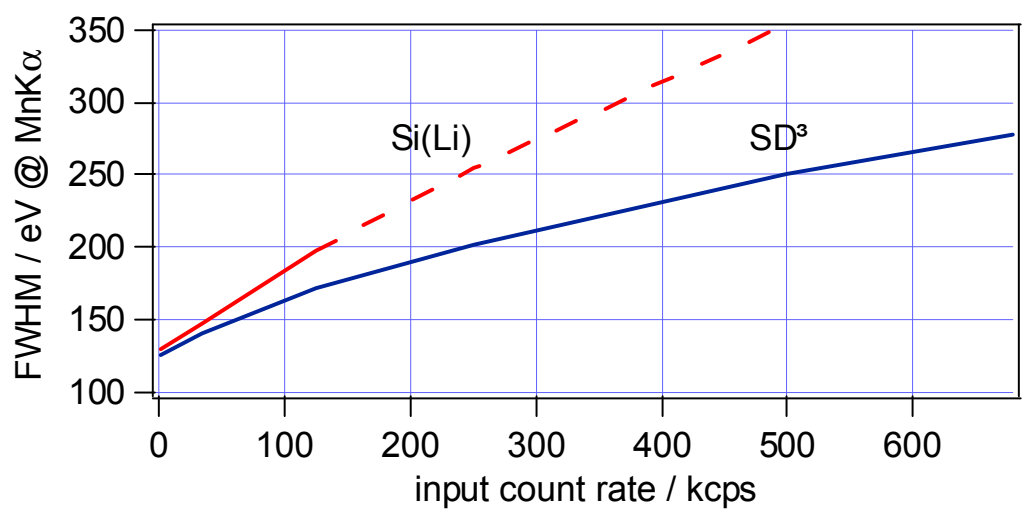

FIG. 3. Energy resolution (FWHM) of $\mathrm{SD}^{3}$ detectors and state-of-the-art $\mathrm{Si}(\mathrm{Li})$
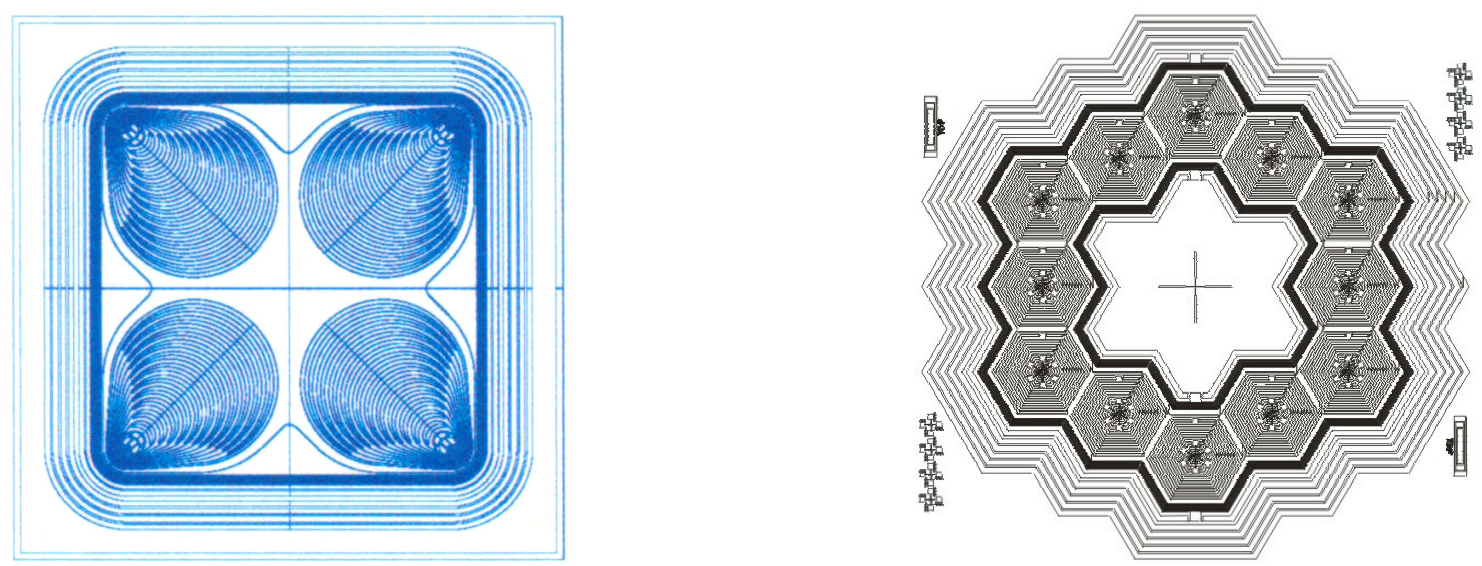

FIG. 4. 4 channel cloverleaf $\mathrm{SD}^{3}$ and annular 12 channel SDD chip 